\title{
Energy transfer and trapping in Synechococcus WH 7803
}

\author{
Alonso M. Acuña ${ }^{1}$ Claire Lemaire $^{2} \cdot$ Rienk van Grondelle $^{1} \cdot$ Bruno Robert $^{1,2}$. \\ Ivo H. M. van Stokkum ${ }^{1}$ (1)
}

Received: 21 June 2017 / Accepted: 26 September 2017 / Published online: 13 October 2017

(C) The Author(s) 2017. This article is an open access publication

\begin{abstract}
Excitation energy transfer (EET) and trapping in Synechococcus WH 7803 whole cells and isolated photosystem I (PSI) complexes have been studied by timeresolved emission spectroscopy at room temperature (RT) and at $77 \mathrm{~K}$. With the help of global and target analysis, the pathways of EET and the charge separation dynamics have been identified. Energy absorbed in the phycobilisome (PB) rods by the abundant phycoerythrin (PE) is funneled to phycocyanin (PC645) and from there to the core that contains allophycocyanin (APC660 and APC680). Intra-PB EET rates have been estimated to range from 11 to $68 / \mathrm{ns}$. It was estimated that at RT, the terminal emitter of the phycobilisome, APC680, transfers its energy at a rate of 90/ns to PSI and at a rate of 50/ns to PSII. At $77 \mathrm{~K}$, the redshifted $\mathrm{Chl} a$ states in the PSI core were heterogeneous, with maximum emission at 697 and $707 \mathrm{~nm}$. In $72 \%$ of the PSI complexes, the bulk Chl $a$ in equilibrium with F697 decayed with a main trapping lifetime of $39 \mathrm{ps}$.
\end{abstract}

Keywords Excitation energy transfer - Global analysis . Light harvesting $\cdot$ Target analysis

Electronic supplementary material The online version of this article (doi:10.1007/s11120-017-0451-2) contains supplementary material, which is available to authorized users.

Ivo H. M. van Stokkum

i.h.m.van.stokkum@vu.nl

1 Faculty of Science, Department of Physics and Astronomy and Institute for Lasers, Life and Biophotonics, Vrije Universiteit, Amsterdam, Netherlands

2 CEA, Institut de Biologie et de Technologies de Saclay, and CNRS, 91191 Gif/Yvette Cedex, France

\author{
Abbreviations \\ APC Allophycocyanin \\ DAS Decay-associated spectrum \\ EAS Evolution-associated spectrum \\ EET Excitation energy transfer \\ FWHM Full width at half maximum \\ IRF Instrument response function \\ PB Phycobilisome \\ PC Phycocyanin \\ PE Phycoerythrin \\ PS Photosystem \\ PU Phycourobilin \\ RP Radical pair \\ rms Root mean square \\ SAS Species-associated spectrum \\ SNR Signal to noise ratio \\ SVD Singular value decomposition \\ TRES Time-resolved emission spectrum
}

\section{Introduction}

Photosynthesis is key to the conversion of solar energy to biomass. Light-harvesting antennae absorb sunlight and transfer the excitation energy ultimately to the reaction centers (RCs). The phycobilisome (PB) is the light-harvesting antenna of many cyanobacteria, red algae and glaucophytes (Adir 2005; Glazer 1984; Watanabe and Ikeuchi 2013). Light is absorbed by phycocyanobilin pigments that are covalently bound to phycobiliproteins (Glazer 1984). Synechococcus are marine cyanobacteria that are estimated to assimilate $8 \mathrm{Gt} \mathrm{C} /$ year, corresponding to $\approx 17 \%$ of the ocean net primary production (Flombaum et al. 2013). In Synechococcus WH7803 (Waterbury et al. 1986), the rods consist of three types of hexamers, which are named after 
Table 1 Absorption and emission maxima of the rod hexamers according to Ong and Glazer $(1987,1991)$ and Six et al. (2007)

\begin{tabular}{lll}
\hline Hexamer & $\mathrm{A}_{\max }(\mathrm{nm})$ & $\mathrm{F}_{\max }(\mathrm{nm})$ \\
\hline PEII & $544(\mathrm{PEII}), \mathrm{sh} 498(\mathrm{PU})$ & 563 \\
PEI & $550(\mathrm{PEI})$ & 572 \\
R-PCII & $533,554(\mathrm{PE}), 615(\mathrm{PC})$ & 646 \\
\hline
\end{tabular}

sh shoulder

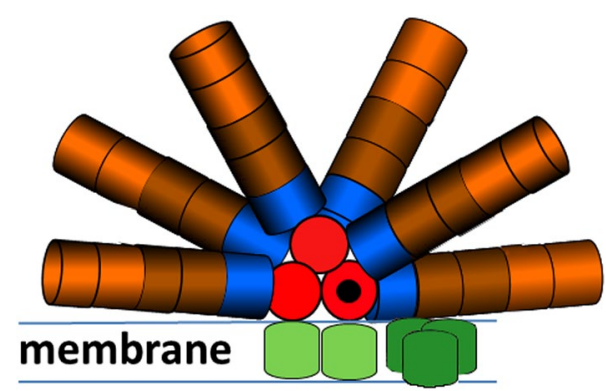

Fig. 1 Cartoon model for the Synechococcus WH7803 PB structure proposed by Six et al. (2007) combined with the megacomplex structure of Liu et al. (2013). Key: PEII (brown), PEI (maroon), R-PCII (blue), APC660 (red), APC680 (black), PSII dimer (green) and PSI trimer (dark green)

the phycocyanin (PC) and phycoerythrin (PE) pigments they contain. A model for the PB structure has been proposed by Six et al. (2007). From tip to core, each rod consists of five hexamers: two PEII, two PEI and one R-PCII. In addition to $\mathrm{PE}$, the PEII hexamer contains phycourobilin (PU) as well in a PE:PU ratio of 5:1 (Six et al. 2007). Six rods radiate from a core consisting of three cylinders that contain allophycocyanin (APC) pigments. This pattern of three cylinders and six rods is analogous to that of PBs of Synechocystis sp. PCC 6803 (Arteni et al. 2009). The spectral properties of the rod hexamers (Ong and Glazer 1987, 1991; Six et al. 2007) are summarized in Table 1.

Together, these PE, PC and APC pigments absorb light between 400 and $650 \mathrm{~nm}$. Excitations of the PB pigments are efficiently transferred to the chlorophyll-containing photosystems (PS) I and II (Tian et al. 2011, 2012, 2013b; Scott et al. 2006; Gillbro et al. 1985; Sandstrom et al. 1988; Liu et al. 2013; Dong et al. 2009). A cartoon model for the structure of PB, assuming that PB can transfer to both PSI and PSII (Liu et al. 2013) is sketched in Fig. 1.

These photosystems convert the excitations to chemical energy via initial charge separation (van Grondelle et al. 1994), and the combined action of PBs and photosystems (the light reactions of photosynthesis) provides the energy input to the cell. Excitation energy transfer (EET) and trapping have recently been described in detail in a PSI-deficient mutant of Synechocystis sp. PCC 6803 (Acuña et al. 2017).
By means of a target analysis (Holzwarth 1996; van Stokkum et al. 2004), it was estimated that the terminal emitter of the phycobilisome, termed APC680, transfers its energy at a rate of $(20 \mathrm{ps})^{-1}$ to PSII. This is faster than the intraphycobilisome EET rates between a rod and a core cylinder, or between the core cylinders (van Stokkum et al. 2017). Here, we measure ultrafast time-resolved emission spectra in whole cells of Synechococcus WH7803 at room temperature and at $77 \mathrm{~K}$ to study EET and trapping. With the help of target analysis, we estimate the unknown rates of EET within the Synechococcus WH7803 PB and between the PB and the photosystems.

\section{Materials and Methods}

\section{Growth conditions and sample preparation}

\section{Cell culture and growth conditions}

Synechococcus sp. strain WH 7803 (from the Woods Holes Oceanographic Institution, USA) (Waterbury et al. 1986) was grown at the CEA (Saclay, FR) in an artificial seawater medium (Wyman et al. 1985) at $20^{\circ} \mathrm{C}$ with $10 \mu \mathrm{mol}$ photons $\mathrm{m}^{-2} \mathrm{~s}^{-1}$. The experimental cultures were continuously bubbled with sterile air.

\section{Sample handling}

Samples of the main batch were then taken to the LaserLab (Amsterdam, NL) and cooled in dry ice and in darkness to perform time-resolved fluorescence measurements. Before the first measurement, the cells were first acclimated for ca. $20 \mathrm{~min}$ to $40 \mu \mathrm{mol}$ photons $\mathrm{m}^{-2} \mathrm{~s}^{-1}$ in an Erlenmeyer flask continuously shaken at $250 \mathrm{rpm}$. The flask was kept in those conditions. For each time range, a new sample was taken from the Erlenmeyer flask. Streak images were all acquired within the first $48 \mathrm{~h}$ after arrival of the sample at the LaserLab in Amsterdam.

\section{Thylakoid extraction and PSI isolation}

Thylakoid extraction was performed as described previously (Post et al. 1992). PSI was purified by sucrose density gradient centrifugation as described previously (Marquardt and Rhiel 1997).

\section{Steady-state absorption}

Steady-state absorption of whole cells was measured using a Varian Cary 4000 UV-Vis spectrophotometer additionally equipped with a Varian 900 external diffuse reflectance accessory. 


\section{Time-resolved fluorescence}

A series of streak camera measurements (Van Stokkum et al. 2008; Wlodarczyk et al. 2016), comprising several image sequences using different parameters, was carried out less than $1 \mathrm{~h}$ after taking the cells out of the flask to ensure excellent sample quality. While there is no evidence for significant cell degradation during single sequences, the cells may have changed from one sequence to the next. The different conditions from one sequence to another include two different excitation wavelengths $(400 \mathrm{~nm}$ for predominant $\mathrm{Chl}$ excitation; $550 \mathrm{~nm}$ for predominant $\mathrm{PE}$ excitation), or different time ranges (TR): from 0 to $400 \mathrm{ps}$ (TR2) and from 0 to 1500 ps (TR4). For measurements performed using TR2 (TR4), the image sequence consists of 300 (150) images, each of which results from a scan of $8 \mathrm{~s}$. To achieve a high SNR, each image sequence is used to produce an average image that is, in turn, corrected for background and lamp shading before analysis. To judge whether the sample changed over time, we kept track of the chronological order in sequence acquisition. We indicate the conditions as 'time range/ $\lambda_{\mathrm{exc}}$ (in $\mathrm{nm}$ )'. Measurements were done at room temperature (RT) and at $77 \mathrm{~K}$. At RT, measurements were carried out with two batches $\mathrm{A}$ and B. In batch $\mathrm{A}$, the laser power used was $45 \mu \mathrm{W}$ and the acquisition order was: TR2/400 $\rightarrow$ TR4/400 $\rightarrow$ $\mathrm{TR} 4 / 550 \rightarrow \mathrm{TR} 2 / 550$. In Batch B, the laser power was 60 $\mu \mathrm{W}$ and the acquisition order was: TR2/400 $\rightarrow$ TR4/400 $\rightarrow$ TR2/550 $\rightarrow$ TR4/550. At $77 \mathrm{~K}$, the laser power used was $15 \mu \mathrm{W}$ and the acquisition order was: TR4/550 $\rightarrow$ $\mathrm{TR} 2 / 550 \rightarrow \mathrm{TR} 2 / 400 \rightarrow \mathrm{TR} 4 / 400$. To avoid annihilation, the laser power was at most $60 \mu \mathrm{W}$.

The samples frozen to $77 \mathrm{~K}$ in a Pasteur pipette were placed in a cold finger. The optical path length within the sample was $\approx 1 \mathrm{~mm}$. The fluorescence at the angle of $90^{\circ}$ to the direction of the excitation beam was collimated and focused onto the input slit of spectrograph Chromex 250IS (Chromex, Albuquerque, New Mexico). With $550 \mathrm{~nm}$ excitation at RT and with both excitations at $77 \mathrm{~K}$ a cutoff filter OC13 was used to block the scattered excitation light. This filter attenuates light with wavelengths below $620 \mathrm{~nm}$. Spectrally resolved emission was detected using a Hamamatsu C5680 synchroscan camera with a cooled Hamamatsu Digital Camera C10600-10B (ORCA-R2) (Hamamatsu Photonics, Hamamatsu, Japan). In all cases, the laser light was vertically polarized, the spot size was $60 \mu \mathrm{m}$, the laser repetition rate was set to $250 \mathrm{kHz}$, the input slit of the spectrograph was $140 \mu \mathrm{m}$ and that of the photo-cathode of the streak camera was $220 \mu \mathrm{m}$ and the detection was parallel (VV) to the incident polarization. The full width at half maximum (FWHM) of the instrument response function (IRF) was $\approx 10 \mathrm{ps}$ with TR2 and $\approx 25$ ps with TR 4 . At $77 \mathrm{~K}$, The FWHM of the IRF was $\approx 17$ ps with TR 2 and $\approx 27$ ps with TR 4 .
Global and target analysis of time-resolved emission spectra

In target analysis of time-resolved emission spectra, the inverse problem is to determine the number of electronically excited states $\left(N_{\text {states }}\right)$ present in the system, and to estimate their spectral properties $\operatorname{SAS}_{l}(\lambda)$ and their populations $c_{l}^{S}(t)$ (superscript $\mathrm{S}$ stands for species). The time-resolved emission spectra TRES $(t, \lambda)$ are described by a parameterized superposition model:

$\operatorname{TRES}(t, \lambda)=\sum_{l=1}^{N_{\text {states }}} c_{l}^{S}(t, \theta) \operatorname{SAS}_{l}(\lambda)$,

where the populations are determined by an unknown compartmental model that depends upon the unknown kinetic parameters $\theta$. In the target analysis, constraints on the $S A S$ are needed to estimate all parameters $\theta$ and $\operatorname{SAS}_{l}(\lambda)$ (Snellenburg et al. 2013; van Stokkum et al. 2004).

The population of the $l$-th compartment is $c_{l}^{S}(t)$. The concentrations of all compartments are collated in a vector: $c^{S}(t)=\left[\begin{array}{lllll}c_{1}^{S}(t) & c_{2}^{S}(t) & \ldots & c_{n_{\text {comp }}}^{S}(t)\end{array}\right]^{T}$ which obeys the differential equation

$\frac{d}{d t} c^{S}(t)=K c^{S}(t)+j(t)$

where the transfer matrix $K$ contains off-diagonal elements $k_{p q}$, representing the microscopic rate constant for EET from compartment $q$ to compartment $p$. The diagonal elements contain the total decay rates of each compartment. The input to the compartments is $j(t)=\operatorname{IRF}(t)\left[\begin{array}{lll}x_{1} & \ldots & x_{n_{\text {comp }}}\end{array}\right]^{T}$, with $x_{l}$ the absorption of the $l$-th compartment.

The impulse response of the system, which is a sum of exponential decays, has to be convolved with the IRF. Typically, a Gaussian-shaped IRF is adequate, with parameters $\mu$ for the location of the IRF maximum and $\Delta$ for the FWHM of the IRF:

$\operatorname{IRF}(t)=\frac{1}{\tilde{\Delta} \sqrt{2 \pi}} \exp \left(-\log (2)(2(t-\mu) / \Delta)^{2}\right)$

where $\tilde{\Delta}=\Delta /(2 \sqrt{2 \log (2)})$. The convolution (indicated by an *) of this IRF with an exponential decay (with decay rate $k$ ) yields an analytical expression which facilitates the estimation of the decay rate $k$ and the IRF parameters $\mu$ and $\Delta$ :

$$
\begin{gathered}
c^{D}(t, k, \mu, \Delta)=\exp (-k t) * \operatorname{IRF}(t) \\
\quad=\frac{1}{2} \exp (-k t) \exp \left(k\left(\mu+\frac{k \tilde{\Delta}^{2}}{2}\right)\right) \\
\left\{1+\operatorname{erf}\left(\frac{t-\left(\mu+k \tilde{\Delta}^{2}\right)}{\sqrt{2} \tilde{\Delta}}\right)\right\} .
\end{gathered}
$$


Typically, with streak camera measurements, the IRF can be well approximated by a sum of up to three Gaussians.

The solution of the general compartmental model described by the $K$ matrix consists of exponential decays with decay rates equal to the eigenvalues of the $K$ matrix. When the compartmental model consists of independently decaying species, their spectra are termed $\operatorname{DAS}_{l}(\lambda)$ (decayassociated spectra), and when it consists of a sequential scheme with increasing lifetimes the spectra are termed $\operatorname{EAS}_{l}(\lambda)$ (evolution-associated spectra).

The interrelation between the DAS and SAS is expressed in the following matrix equation:

$C^{D}(\theta, \mu, \Delta) \cdot \operatorname{DAS}^{T}=C^{S}(\theta, \mu, \Delta) \cdot \mathrm{SAS}^{T}$.

Here, the matrix $C^{D}(\theta, \mu, \Delta)$ contains in its $l$-th column the decay $c_{l}^{D}\left(t, k_{l}, \mu, \Delta\right)$ and the matrix $C^{S}(\theta, \mu, \Delta)$ contains in its columns the populations $c_{l}^{S}(t)$ of the general compartmental model.

\section{Simultaneous target analysis}

To resolve the different species and to improve the precision of the estimated parameters, the set of $N_{\text {exp }}$ experiments that describe the same sample (measured with different excitation wavelengths or on different time ranges) can be analyzed simultaneously. For each additional data set $\mathrm{TRS}_{e}$ one scaling parameter $\alpha_{e}$ and one time shift parameter $\mu_{e}$ must be added:

$\operatorname{TRS}_{e}=\alpha_{e}\left(C_{e}^{S}\left(\theta, \mu_{e}, \Delta\right) \cdot \mathrm{SAS}^{T}\right.$.

The different excitation wavelengths are taken into account via the absorptions of the species that result in $C_{e}^{S}\left(\theta, \mu_{e}, \Delta\right)$

\section{Residual analysis}

Following a successfully converged fit, the matrix of residuals is analyzed with the help of a singular value decomposition (SVD). Formally, the residual matrix can be decomposed as

$\operatorname{res}(t, \lambda)=\sum_{l=1}^{m} u_{l}^{\mathrm{res}}(t) s_{l} w_{l}^{\mathrm{res}}(\lambda)$,

where $u_{l}$ and $w_{l}$ are the left and right singular vectors, $s_{l}$ the sorted singular values, and $m$ is the minimum of the number of rows and columns of the matrix. The singular vectors are orthogonal and provide an optimal least squares approximation of the matrix. The SVD of the matrix of residuals is useful to diagnose the shortcomings of the model used or systematic errors in the data.

\section{Results and discussion}

\section{Measurements at room temperature}

The RT absorption spectrum of Synechococcus WH7803 (black in Fig. 2) is dominated by the PE absorption around $550 \mathrm{~nm}$. The absorption spectra of the three hexamers [taken from Six et al. (2007) and Ong and Glazer (1987)] are consistent with this dominant PE absorption. The absorption maximum is most close to that of PEII (brown).

At RT, a global analysis requires five or six lifetimes with 550 or $400 \mathrm{~nm}$ excitation, cf. Fig. 3 and Table 2. The EAS estimated with $550 \mathrm{~nm}$ excitation (Fig. 3a) is attenuated on the blue side because a cutoff filter had to be used to suppress the scattered excitation light. The emission below $600 \mathrm{~nm}$ can be attributed to PEI and PEII. The decay is multiexponential, with rise components present in the cyan and black DAS with $400 \mathrm{~nm}$ excitation (Fig. 3d). The first two lifetimes with $550 \mathrm{~nm}$ excitation are both $30 \mathrm{ps}$; thus, their DAS are compensating and cannot be plotted. A clear redshift of the PE emission is visible going from the black to the red EAS (Fig. 3a, c). After $400 \mathrm{~nm}$ excitation, in the Chl $a$ emission region $(670-700 \mathrm{~nm})$, PSI equilibrates with 8.5 ps and then decays with 22 ps (cyan and black EAS in Fig. 3c). The final DAS of $\approx 1360$ ps (magenta) can be interpreted as a small fraction of non-transferring PB (peaking at $\approx 677 \mathrm{~nm})$ and a small fraction of non-transferring $\mathrm{PE}$ (peaking at $\approx 570 \mathrm{~nm})$. The green DAS $(\approx 130 \mathrm{ps})$ also shows two peaks (Fig. 3b, d). The largest peak (at $\approx 677 \mathrm{~nm}$ ) can be attributed to trapping in the photosystems. The blue DAS $(\approx 70 \mathrm{ps})$ is almost conservative in Fig. $3 \mathrm{~b}$, and can be attributed to EET from PB to the photosystems. These

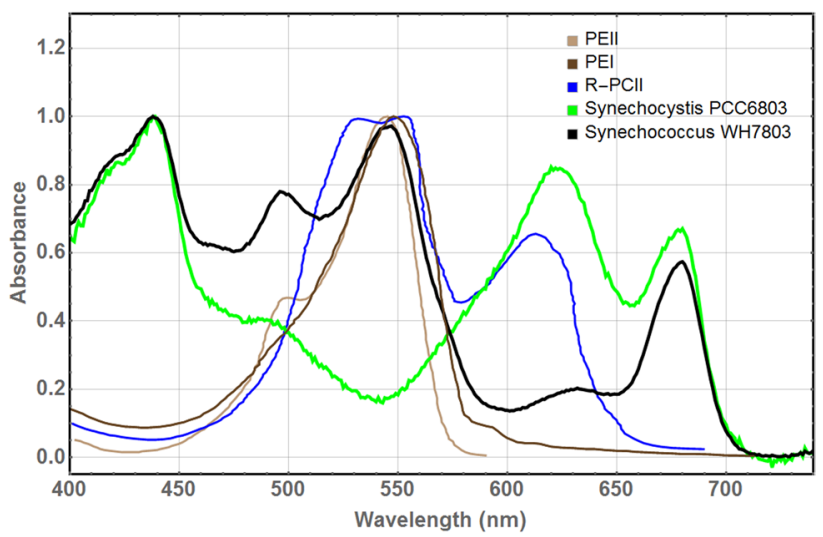

Fig. 2 Normalized RT absorption spectra of whole cells of cyanobacterium Synechococcus WH7803 (black) and Synechocystis PCC6803 (green) in comparison with the spectra of PEII (maroon) and PEI (brown) complexes isolated from Synechococcus WH7803 (Six et al. 2007) and R-PCII (blue) in $0.05 \mathrm{M}$ sodium phosphate buffer (Ong and Glazer 1987) 
A
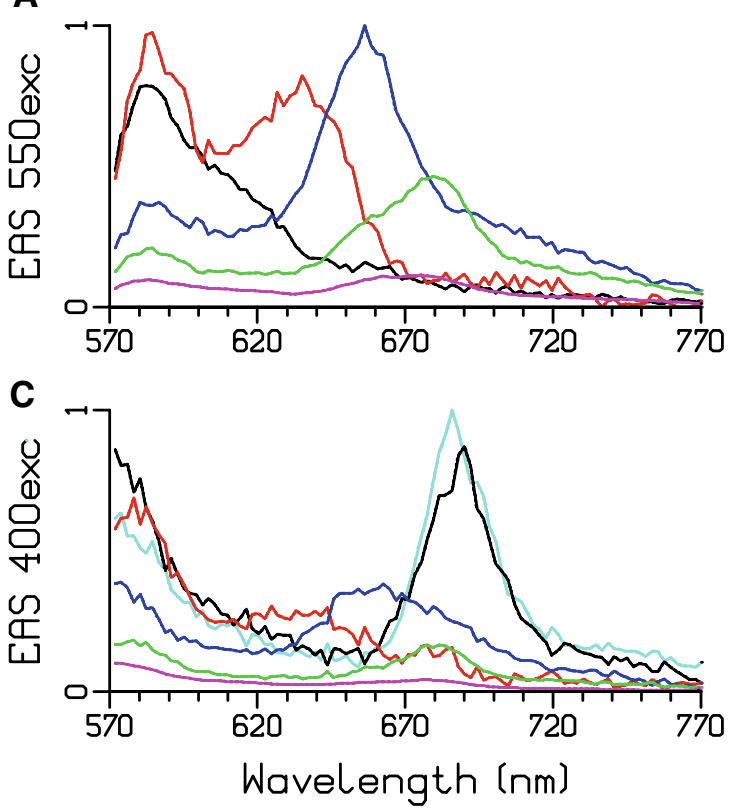

B
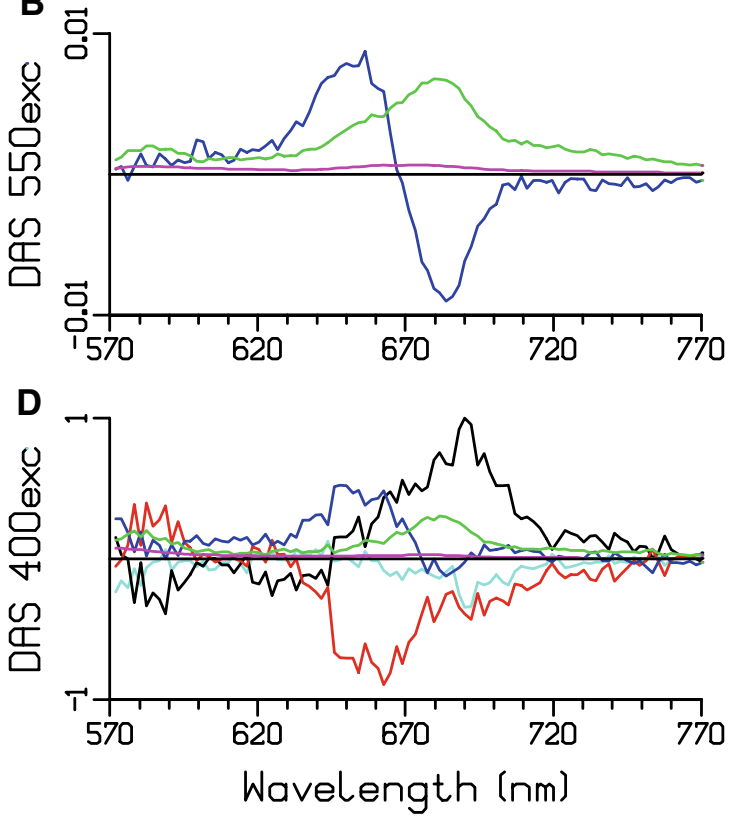

Fig. 3 Estimated EAS and DAS after $550(\mathbf{a}, \mathbf{b})$ or 400 (c, d) nm excitation of whole cells of Synechococcus WH7803 at RT. Estimated lifetimes are collated in Table 2

Table 2 Estimated lifetimes (in ps) after 550 or $400 \mathrm{~nm}$ excitation of whole cells of Synechococcus WH7803 at RT

\begin{tabular}{lllllll}
\hline$\lambda_{\text {exc }}(\mathrm{nm})$ & $\tau 1$ (cyan) & $\tau 2$ (black) & $\tau 3$ (red) & $\tau 4$ (blue) & $\tau 5$ (green) & $\tau 6$ (magenta) \\
\hline 550 & - & 30 & 30 & 63 & 118 & 1374 \\
400 & 8.5 & 22 & 43 & 79 & 139 & 1351 \\
\hline
\end{tabular}

Table 3 Estimated fractions of the different complexes in the experiments at RT with 400 and $550 \mathrm{~nm}$ excitation

\begin{tabular}{lcc}
\hline & 400 exc $(\%)$ & 550 exc $(\%)$ \\
\hline PB-PSII-PSI & 80 & 79 \\
Non-transferring PB & 9 & 14 \\
Non-transferring PE & 10 & 7 \\
\hline
\end{tabular}

complicated DAS can only be further interpreted with the help of a target analysis.

Our target model consists of three different complexes: a PB-PSII-PSI complex with PSII RCs open (estimated to be $79 \%$ with $550 \mathrm{~nm}$ excitation), non-transferring PB (14\%) and non-transferring PE (7\%) (Table 3).

In a simultaneous target analysis of the four data sets collected with 400 and $550 \mathrm{~nm}$ excitation and two different time ranges, we linked all SAS, except for the PE SAS which were allowed to differ below $653 \mathrm{~nm}$ because of the attenuation on the blue side by the cutoff filter used with $550 \mathrm{~nm}$ excitation. An important distinction between PSI and PSII is the rate of trapping. In Synechococcus WH 7803 PSI the major trapping lifetime in vitro was $18 \mathrm{ps}$, with some trapping and equilibration with a redshifted $\mathrm{Chl}$ in $7.5 \mathrm{ps}$ (van Stokkum et al. 2013). Thus, the biexponential decay of the PSI emission can be described with two compartments for bulk and redshifted Chl that equilibrate. Trapping is modelled with a decay rate of $80 / \mathrm{ns}$ from the bulk Chl. In these experiments, one cannot distinguish between different models for EET and trapping in PSII (Holzwarth et al. 2006; Raszewski and Renger 2008). The biexponential decay of the PSII dimer emission (Tian et al. 2013a) has been described by an equilibrium of the Chl $a$ compartment with a radical pair (RP) compartment. The rate from the PSII Chl $a$ to RP was estimated to be $14 / \mathrm{ns}$. This large difference in the trapping dynamics between PSI and PSII allows estimation of the rates of EET from PB to PSI or PSII. When after $550 \mathrm{~nm}$ excitation and EET the Chl emission ultimately decays with a rate much faster than $14 / \mathrm{ns}$, it can be concluded that an appreciable amount of the PB excitations is quenched by trapping in PSI. The EET rate to PSII was estimated to be $50 / \mathrm{ns}$, which is the same as the value estimated by Acuña et al. (2017) in a target analysis of whole cells of a PSI-deficient mutant of Synechocystis sp. PCC 6803. The estimated relative precision of each estimated rate constant was $20 \%$. The most striking finding of the estimated rates 
in Fig. 4 is that the EET rate to PSI is larger than that to PSII. This is in agreement with the efficient EET (estimated quantum efficiency up to 0.89 ) from PB to PSI determined in Synechococcus sp. PCC 7002 (Dong et al. 2009).

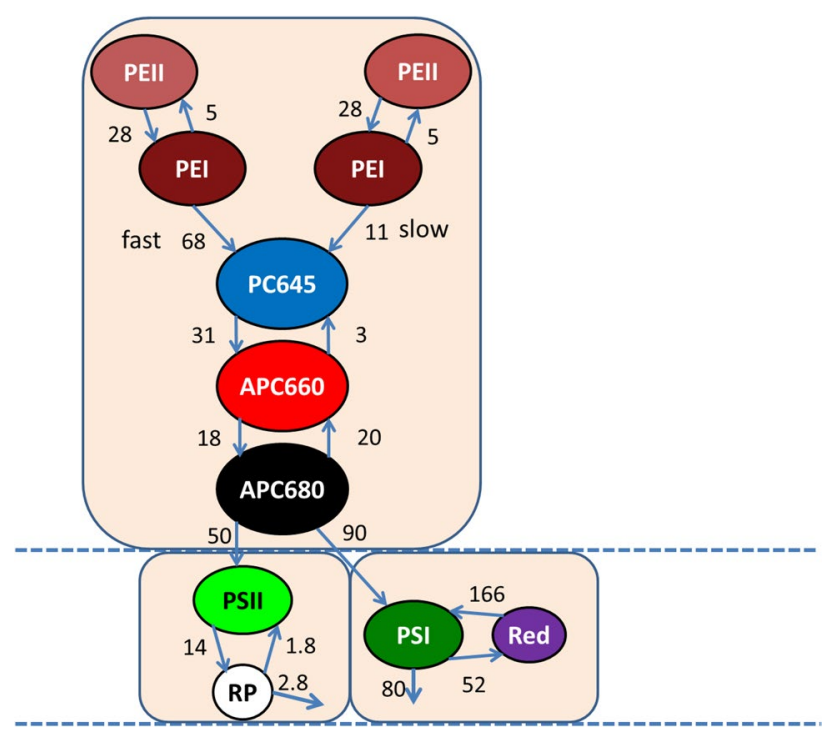

Fig. 4 Functional compartmental model of the PB-PSII-PSI complex with RCs open at RT. All microscopic rate constants are in $1 / \mathrm{ns}$. The common $k_{f l}$ rate constant for excited PE, PC, APC, PSII and red states of $0.86 /$ ns has been omitted for clarity
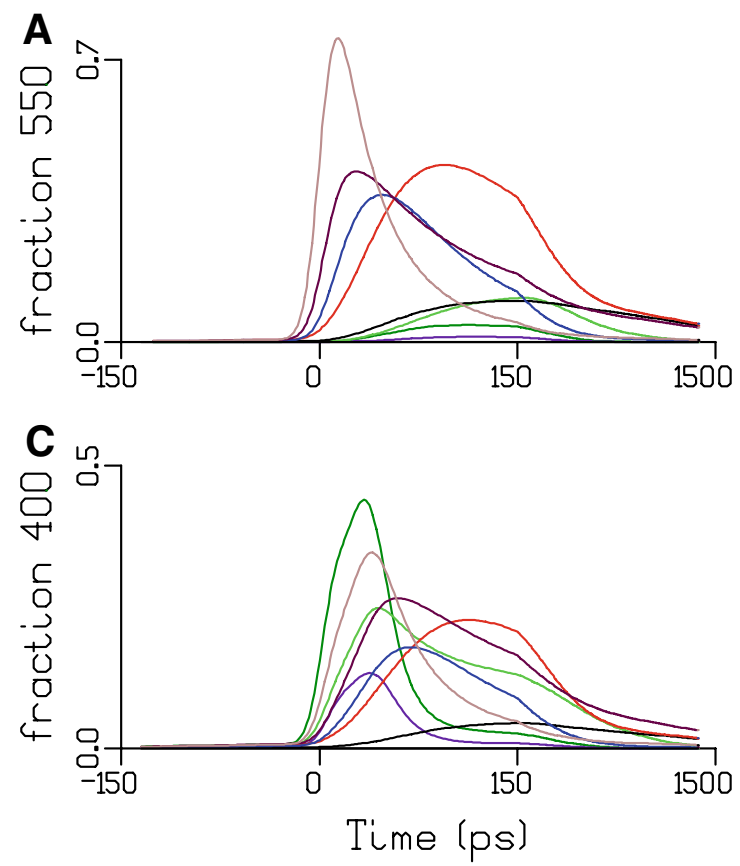

The EET rate between PE and PC645 is heterogeneous. The fast fraction (68/ns) is 73 or $62 \%$ with 550 or $400 \mathrm{~nm}$ excitation. The remainder ( 27 or $38 \%$ ) transfers with a slow rate of 11 or $9 / \mathrm{ns}$ with 550 or $400 \mathrm{~nm}$ excitation. The fast fraction can be attributed to the PE pigments that are most close to the PC645 pigments, which are located in the PC hexamer (Ong and Glazer 1987) and in the neighbouring PEI hexamer. The slow fraction can be attributed to the PE pigments more distal to the core. The fast rate is similar to the intrahexamer EET rate of $69 / \mathrm{ns}$, whereas the slow rate is very close to the effective rod to core EET rate of 12/ ns that were both estimated in Synechocystis (Acuña et al. 2017; van Stokkum et al. 2017). The estimated EET rates are consistent with calculations of Förster EET rates in rods (Xie et al. 2002).

In panels $\mathrm{A}$ and $\mathrm{C}$ of Fig. 5, the total concentration is plotted. For each species, the total concentration is the sum of all excited state populations in the compartments with the spectrum of that species. Note that the initial population of PSI and PSII Chl $a$ greatly increases with $400 \mathrm{~nm}$ excitation (Fig. 5c), which is absorbed well due to the Soret band of $\mathrm{Chl} a$. The percentages excitation of each pigment type are collated in Table 4 and are consistent with the properties of the six types of pigments. Note that APC680 and PSI "Red Chl" are not in Table 4, since the amount of these excited pigments is very small compared to APC660 and bulk PSI $\mathrm{Chl} a$. The time zero spectrum is the weighted sum of the SAS of all the excited pigments. Thus, the shape of the SAS
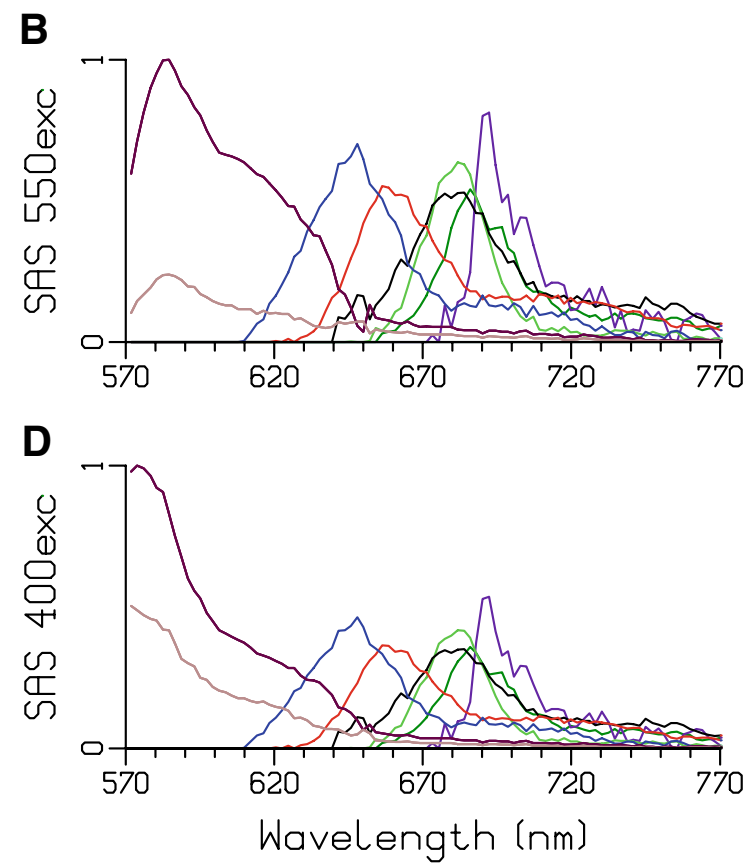

Fig. 5 Total concentrations and SAS estimated after 550 (a, b) or 400 (c, d) nm exc. Key: PEII (brown), PEI (maroon), PC645 (blue), APC660 (red), APC680 (black), PSII Chl $a$ (green), PSI Chl $a$ (dark green) and PSI "Red Chl" (purple) 
Table 4 Percentage excitation of each pigment type with the different excitation wavelengths at RT

\begin{tabular}{lcl}
\hline & 400 exc $(\%)$ & 550 exc $(\%)$ \\
\hline PEI & 3.5 & 16.3 \\
PEII & 21.1 & 79.7 \\
PC645 & 1.5 & 1.9 \\
APC660 & 1.1 & 2.0 \\
PSI & 61.7 & \\
PSII & 11.0 & \\
\hline
\end{tabular}

of the fastest decaying species, PEI, is the most sensitive to the relative absorption parameters. The parameters of Table 4 that led to the acceptable SAS of Fig. 5 were thus determined iteratively.

The estimated SAS depicted in Fig. 5d are consistent with the properties of the eight types of pigments. The maxima of the emission are at $<570,574,646,658,663,684,682,686$, and $690 \mathrm{~nm}$ for, respectively, PEII (brown), PEI (maroon), PC645 (blue), APC660 (red), APC680 (black), PSII Chl $a$ (green), PSI Chl $a$ (dark green) and PSI "Red Chl” (purple). The fit quality of the target analysis is good, cf. Figure S1 and Figure S2. Taking into account the large overlap of the SAS, in particular from 640 to $700 \mathrm{~nm}$, it is remarkable that the SAS have been so nicely resolved. Thus, the simple energy funnel type of kinetic scheme of Fig. 4 allows to resolve the complete EET dynamics. Note that all estimated rate constants represent effective rates, and when more would be known about the structure, it would be possible to infer e.g. the EET rates between the APC660 pigments within and between the three core cylinders (van Stokkum et al. 2017).

The estimates for the PSI equilibration are somewhat faster than the rates estimated in vitro (van Stokkum et al. 2013), whereas the estimated trapping rates are very similar in vivo and in vitro, i.e. 80 and $85 / \mathrm{ns}$. The SAS of the "Red Chl" in PSI is shifted by only $4 \mathrm{~nm}$ relative to the bulk (690 vs. $686 \mathrm{~nm}$, purple vs. dark green in Fig. 5). In agreement with the in vitro results (van Stokkum et al. 2013), the equilibrium favours the bulk Chl $a$ (Fig. 4), which is in contrast to most other cyanobacteria where the "Red Chl" in PSI emits at 708-740 nm (Gobets et al. 2001).

\section{Measurements at $77 \mathrm{~K}$}

At $77 \mathrm{~K}$, a global analysis of the whole cells requires five lifetimes with 550 or $400 \mathrm{~nm}$ excitation, cf. Fig. 6a-d and Table 5. All EAS (and DAS) are attenuated on the blue side, because a cutoff filter had to be used to suppress the scattered excitation light. The emission below $600 \mathrm{~nm}$ can be attributed to PEI and PEII. The PE decay is again strongly multiexponential. With $550 \mathrm{~nm}$ excitation, the DAS of the three longest lifetimes below $600 \mathrm{~nm}$ indicate slow EET with 98 and 470 ps (blue and green DAS in Fig. 6b). In the final DAS of $\approx 3 \mathrm{~ns}$ (magenta in Fig. $6 \mathrm{~b}$ ), the peak at $\approx 590 \mathrm{~nm}$ can be attributed to a small fraction of non-transferring PE, whereas the peak at $\approx 677 \mathrm{~nm}$ can be interpreted as a small fraction of non-transferring PB. The first two lifetimes with $550 \mathrm{~nm}$ excitation are both almost $23 \mathrm{ps}$; thus, their DAS are compensating and cannot be plotted. A clear redshift of the emission is visible going from the black to the red EAS (Fig. 6a), indicating EET. With $400 \mathrm{~nm}$ excitation, in the Chl $a$ emission region (670-700 nm), PSI equilibrates and partly decays with 11 ps (black EAS and DAS in Fig. 6c, d). With both excitation wavelengths, the green DAS $(\approx 400 \mathrm{ps})$ shows three peaks (Fig. $6 \mathrm{~b}, \mathrm{~d}$ ). The peak at $\approx 685 \mathrm{~nm}$ can be attributed to decay of low energy states in the photosystems. The peak at $\approx 685 \mathrm{~nm}$ of the blue DAS ( 98 or $64 \mathrm{ps}$ ) can be attributed to trapping in the photosystems. The peak at $\approx 650 \mathrm{~nm}$ suggests the presence of slowly transferring rods. A target analysis of these $77 \mathrm{~K}$ whole cell data (Fig. 6a-d) is a subject of further research.

A global analysis of a "PSI" sample (with $400 \mathrm{~nm}$ excitation) requires four lifetimes, cf. Fig. 6e, f. The shortest lifetime of $10 \mathrm{ps}$ can be attributed to equilibration (black DAS in Fig. 6f). Next, 31 ps is the main trapping lifetime in PSI (red DAS in Fig. 6f). Both the blue and green DAS (Fig. 6f) show a peak at $\approx 685 \mathrm{~nm}$ that can be attributed to decay in a fraction of PSII that is present in this "PSI" sample. The peak at $\approx 702 \mathrm{~nm}$ in the blue DAS (Fig. 6f) can be attributed to the decay of low energy states in PSI. These complicated DAS can be further interpreted with the help of a target analysis.

Several models exist for PSI and PSII at $77 \mathrm{~K}$ (Snellenburg et al. 2013, 2017; Tian et al. 2013a) which consist of compartments for bulk Chl $a$ in equilibrium with low energy states present in the photosystems. In PSII, at least one, and often two, low energy states are needed. In PSI, the number of low energy states is species dependent. The bulk Chl $a$ decays by trapping (charge separation, photochemical quenching) in the PSI RC and by some quenching process that probably involves formation of a radical pair in the closed PSII RC. The kinetic scheme of Fig. 7 can describe the data of the "PSI" sample, which contained a fraction of PSII. PSII bulk Chl $a$ is in equilibrium with one F686 compartment that represents all the redshifted $\mathrm{Chl} a$ in the CP43 and CP47 core antenna complexes. This equilibrium decays very slowly (4 ns). At $77 \mathrm{~K}$, the heterogeneity of the Red Chl in PSI has to be taken into account (Gobets and van Grondelle 2001). This is modelled by two equilibria between bulk Chl $a$ and a redshifted Chl $a$ compartment. These redshifted Chl $a$ compartments are denoted by their emission maximum F697 and F707. Crucial for resolving the five PS SAS in the target analysis are the spectral constraints. The shape of the vibrational band 

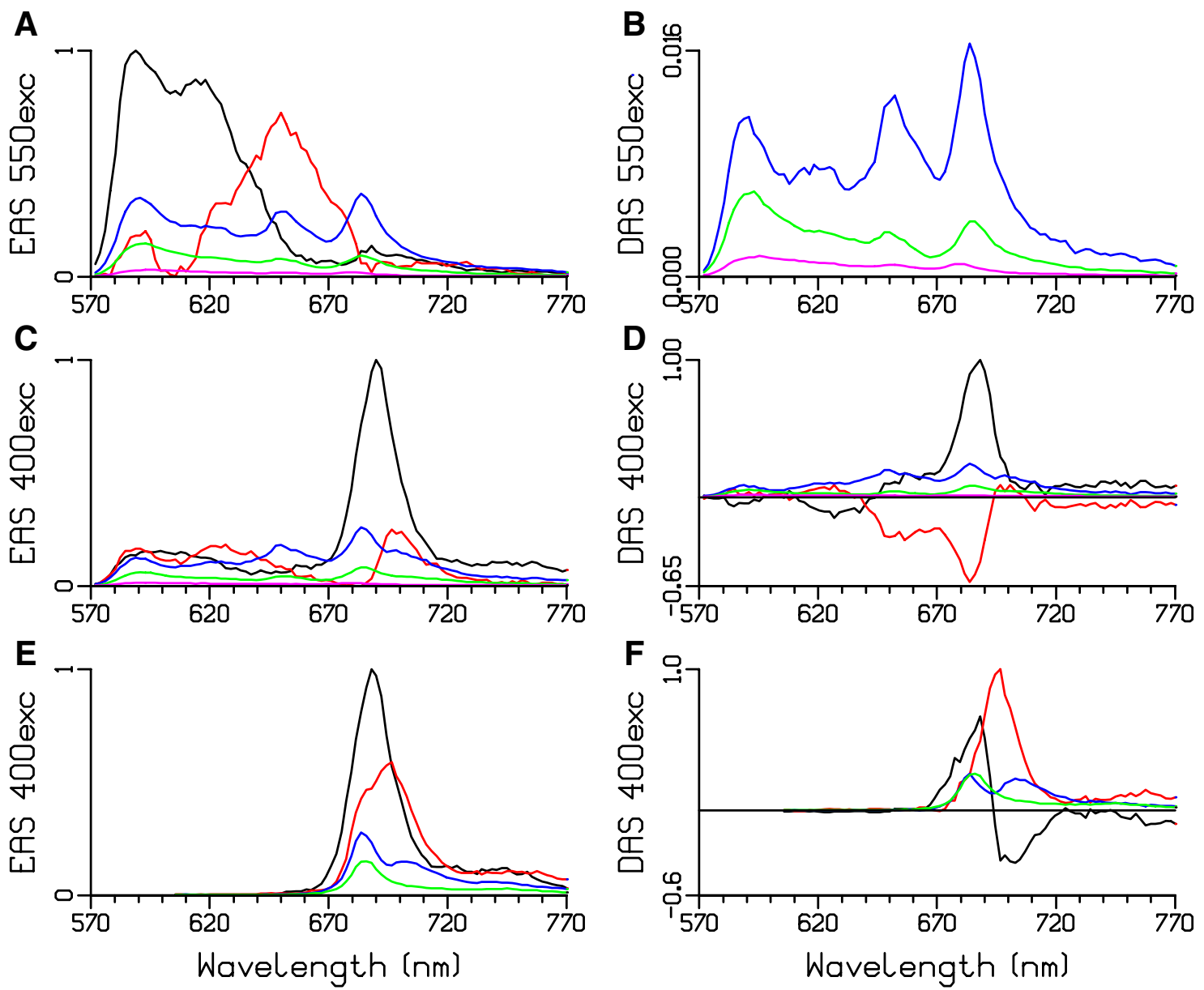

Fig. 6 Estimated EAS and DAS at $77 \mathrm{~K}$ of whole cells after $550(\mathbf{a}, \mathbf{b})$ or $400(\mathbf{c}, \mathbf{d}) \mathrm{nm}$ excitation, and of "PSI" (e, f) after $400 \mathrm{~nm}$ excitation. Estimated lifetimes are collated in Table 5

Table 5 Estimated lifetimes at $77 \mathrm{~K}$ (in ps) of whole cells after 550 or $400 \mathrm{~nm}$ excitation and of "PSI"

Fig. 7 Functional compartmental model of PSI and PSII at $77 \mathrm{~K}$. All microscopic rate constants are in $1 / \mathrm{ns}$. Further explanation is provided in the text

\begin{tabular}{llllccc}
\hline Sample & $\lambda_{\text {exc }}(\mathrm{nm})$ & $\tau 1$ (black) & $\tau 2$ (red) & $\tau 3$ (blue) & $\tau 4$ (green) & $\tau 5$ (magenta) \\
\hline Cell & 550 & 22.6 & 22.9 & 98 & 470 & 2980 \\
Cell & 400 & 11 & 21 & 64 & 317 & 3420 \\
"PSI" & 400 & 10 & 31 & 140 & 1935 & \\
\hline
\end{tabular}
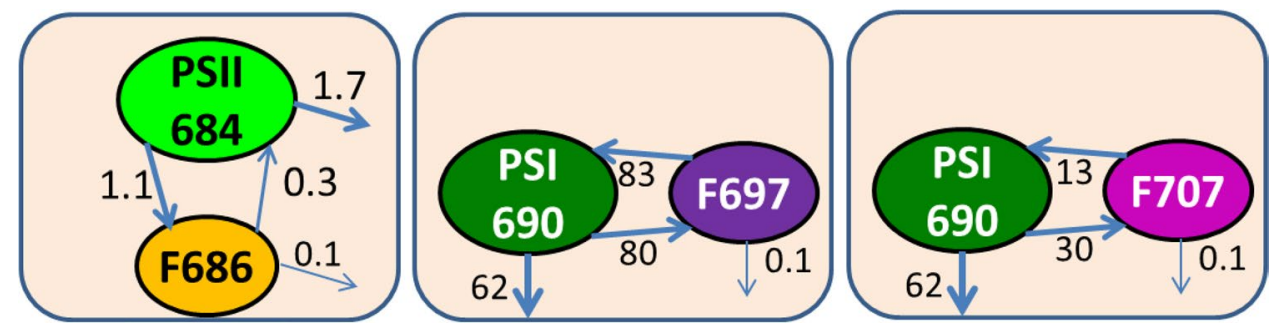

of the two PSII SAS has been assumed to be equal above $690 \mathrm{~nm}$. The SAS of PSI F707 has been assumed to be zero below $695 \mathrm{~nm}$. In addition, to resolve the equilibria, an equal SAS area constraint was instrumental (Snellenburg et al. 2013). The fit quality of the target analysis is excellent (Figure S3).

The populations of the PS compartments are depicted in Fig. 8a, and the amplitude matrix that summarizes their 

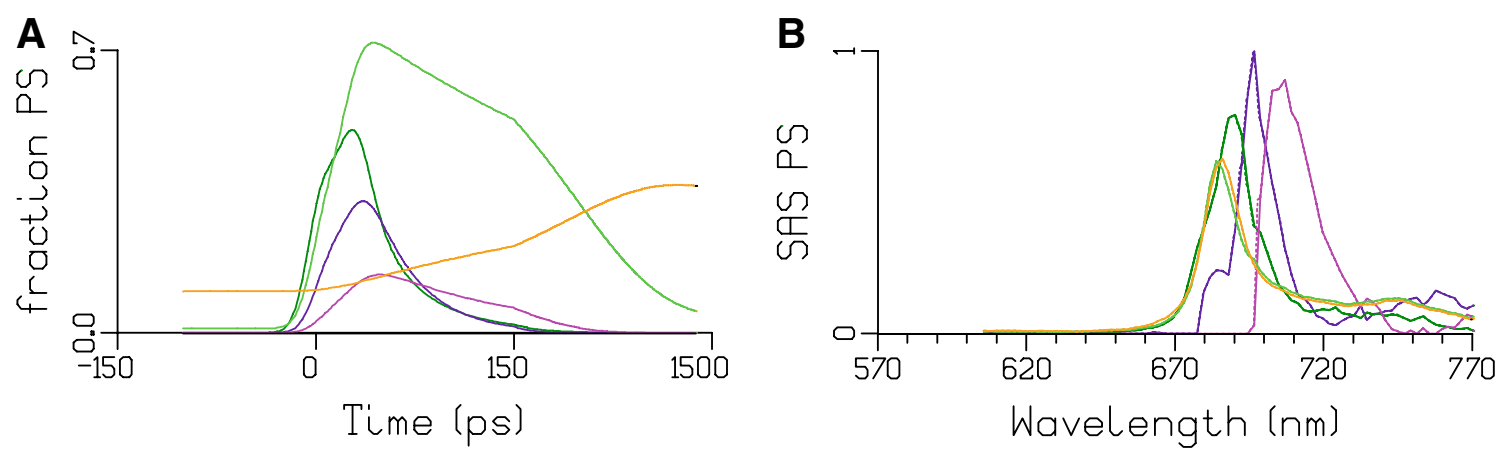

Fig. 8 Total concentrations (a) and SAS (b) estimated after $400 \mathrm{~nm}$ exc of the "PSI" sample. Key: PSII bulk Chl $a$ (green), PSII F686 (orange), PSI bulk Chl $a$ (dark green), PSI F697 (purple) and PSI F707 (magenta)

Table 6 Amplitude matrix of the "PS1" sample at $77 \mathrm{~K}$ with $400 \mathrm{~nm}$ excitation

\begin{tabular}{|r|l|r|r|r|r|r|r|}
\hline Excitation & Species \lifetime(ps) & $\mathbf{5}$ & $\mathbf{1 0}$ & $\mathbf{3 9}$ & $\mathbf{1 2 1}$ & $\mathbf{3 4 1}$ & $\mathbf{4 4 2 2}$ \\
\hline $\mathbf{0 . 5 4}$ & PSI bulk ChI $\boldsymbol{a}$ & 0.36 & 0 & 0.18 & 0 & 0 & 0 \\
\hline 0 & PSI F697 & -0.25 & 0 & 0.25 & 0 & 0 & 0 \\
\hline $\mathbf{0 . 2 1}$ & PSI bulk ChI $\boldsymbol{a}$ & 0 & 0.20 & 0 & 0.01 & 0 & 0 \\
\hline 0 & PSI F707 & 0 & -0.07 & 0 & 0.07 & 0 & 0 \\
\hline $\mathbf{0 . 2 5}$ & PSII bulk Chl $\boldsymbol{a}$ & 0 & 0 & 0 & 0 & 0.24 & 0.01 \\
\hline 0 & PSII F686 & 0 & 0 & 0 & 0 & -0.10 & 0.10 \\
\hline
\end{tabular}

Color code of the species and estimated microscopic rates are given in Fig. 7. Further explanation is provided in the text

dynamics is given in Table 6 . In $(0.54 / 0.75=) 72 \%$ of PSI, the bulk Chl $a$ (dark green) and PSI F697 (purple) equilibrate with a lifetime of $5 \mathrm{ps}$, and this equilibrium decays by trapping with a lifetime of $39 \mathrm{ps}$. In the remaining $28 \%$ of PSI, the bulk Chl $a$ (dark green) and PSI F707 (magenta) equilibrate with a lifetime of $10 \mathrm{ps}$, and this equilibrium decays by delayed trapping with a lifetime of $121 \mathrm{ps}$. Thus, the dominant trapping lifetime in PSI is 39 ps (57\%), and minor trapping lifetimes are $5(15 \%), 10(17 \%)$ and $121 \mathrm{ps}$ (11\%). PSII bulk Chl $a$ (green) and PSII F686 (orange) equilibrate with a lifetime of 341 ps. The PSII emission decays with lifetimes of 341 ps (54\%) and $4.4 \mathrm{~ns}(46 \%)$.

In PSI cores of other cyanobacteria (Gobets et al. 2001), maxima at 708 and $719 \mathrm{~nm}$ were resolved (at RT) in Synechococcus elongatus and Synechocystis sp. PCC 6803, and additionally at $740 \mathrm{~nm}$ in the trimeric core of Spirulina platensis. At RT, these states have a lower free energy than P700, in contrast to the "Red Chl" state in Synechococcus WH 7803 that peaked at $690 \mathrm{~nm}$ (Fig. 5). At $77 \mathrm{~K}$, the inhomogeneity of this "Red Chl" can be modelled by two populations F697 (72\%) and F707 (28\%). F697 can be trapped faster than F707 because the latter has a lower free energy, and the EET to bulk Chl $a$ is smaller. Thus among cyanobacteria, the PSI "Red Chl" in Synechococcus WH 7803, that peak at 697 and $707 \mathrm{~nm}$ at $77 \mathrm{~K}$, are the least redshifted PSI "Red Chl".
Acknowledgements This research was performed as part of the BioSolar Cells research programme, sponsored by the Dutch Ministry of Economic Affairs. This work is part of the research program of the Netherlands Organization for Scientific Research (NWO, previously FOM). BR acknowledges funding from the European Union's Horizon 2020 research and innovation programme under grant agreement no 654148 Laserlab-Europe, project LLAMS002152. IHMvS, BR and RvG acknowledge financial support of the European Research Council (Advanced Grant proposal 267333 (PHOTPROT) to RvG and BR).

Open Access This article is distributed under the terms of the Creative Commons Attribution 4.0 International License (http://creativecommons.org/licenses/by/4.0/), which permits unrestricted use, distribution, and reproduction in any medium, provided you give appropriate credit to the original author(s) and the source, provide a link to the Creative Commons license, and indicate if changes were made.

\section{References}

Acuña AM, van Alphen P, van Grondelle R, van Stokkum IHM (2017) The phycobilisome terminal emitter transfers its energy with a rate of $(20 \mathrm{ps})^{-1}$ to photosystem II. Photosynthetica

Adir N (2005) Elucidation of the molecular structures of components of the phycobilisome: reconstructing a giant. Photosynth Res 85(1): $15-32$

Arteni AA, Ajlani G, Boekema EJ (2009) Structural organisation of phycobilisomes from Synechocystis sp strain PCC6803 and their interaction with the membrane. Biochim Biophys Acta-Bioenerg 1787(4):272-279 
Dong C, Tang A, Zhao J, Mullineaux CW, Shen G, Bryant DA (2009) $\mathrm{ApcD}$ is necessary for efficient energy transfer from phycobilisomes to photosystem I and helps to prevent photoinhibition in the cyanobacterium Synechococcus sp. PCC 7002. Biochim Biophys Acta-Bioenerg 1787(9):1122-1128

Flombaum P, Gallegos JL, Gordillo RA, Rincón J, Zabala LL, Jiao N, Karl DM, Li WKW, Lomas MW, Veneziano D, Vera CS, Vrugt JA, Martiny AC (2013) Present and future global distributions of the marine Cyanobacteria Prochlorococcus and Synechococcus. Proc Natl Acad Sci USA 110(24):9824-9829

Gillbro T, Sandstrom A, Sundstrom V, Wendler J, Holzwarth AR (1985) Picosecond study of energy-transfer kinetics in phycobilisomes of Synechococcus 6301 and the mutant AN 112. Biochim Biophys Acta 808(1):52-65

Glazer AN (1984) Phycobilisome-a macromolecular complex optimized for light energy-transfer. Biochim Biophys Acta 768(1):29-51

Gobets B, van Grondelle R (2001) Energy transfer and trapping in photosystem I. Biochim Biophys Acta (BBA)-Bioenerg 1507(1):80-99

Gobets B, van Stokkum IHM, Rogner M, Kruip J, Schlodder E, Karapetyan NV, Dekker JP, van Grondelle R (2001) Time-resolved fluorescence emission measurements of photosystem I particles of various cyanobacteria: a unified compartmental model. Biophys J 81(1):407-424

Holzwarth AR (1996) Data analysis of time-resolved measurements. In: Amesz J, Hoff AJ (eds) Biophysical techniques in photosynthesis. Kluwer, Dordrecht, pp 75-92

Holzwarth AR, Muller MG, Reus M, Nowaczyk M, Sander J, Rogner M (2006) Kinetics and mechanism of electron transfer in intact photosystem II and in the isolated reaction center: pheophytin is the primary electron acceptor. Proc Natl Acad Sci USA 103(18):6895-6900

Liu H, Zhang H, Niedzwiedzki DM, Prado M, He G, Gross ML, Blankenship RE (2013) Phycobilisomes supply excitations to both photosystems in a megacomplex in cyanobacteria. Science 342(6162):1104-1107

Marquardt J, Rhiel E (1997) The membrane-intrinsic light-harvesting complex of the red alga Galdieria sulphuraria (formerly Cyanidium caldarium): biochemical and immunochemical characterization1. Biochim Biophys Acta(BBA)—Bioenerg 1320(2):153-164

Ong LJ, Glazer AN (1987) R-phycocyanin II, a new phycocyanin occurring in marine Synechococcus species. J Biol Chem 262

Ong LJ, Glazer AN (1991) Phycoerythrins of marine unicellular cyanobacteria. I. Bilin types and locations and energy transfer pathways in Synechococcus spp. phycoerythrins. J Biol Chem 266(15):9515-9527

Post AF, Gal A, Ohad I, Milbauer KM, Bullerjahn GS (1992) Characterization of light-activated reversible phosphorylation of a chlorophyll $\mathrm{a} / \mathrm{b}$ antenna apoprotein in the photosynthetic prokaryote Prochlorothrix hollandica. Biochim Biophys Acta(BBA)-Bioenerg 1100(1):75-82

Raszewski G, Renger T (2008) Light harvesting in photosystem II core complexes is limited by the transfer to the trap: can the core complex turn into a photoprotective mode? J Am Chem Soc 130(13):4431-4446

Sandstrom A, Gillbro T, Sundstrom V, Wendler J, Holzwarth AR (1988) Picosecond study of energy-transfer within 18-S particles of AN-112 (a mutant of Synechococcus 6301) phycobilisomes. Biochim Biophys Acta 933(1):54-64

Scott M, McCollum C, Vasil'ev S, Crozier C, Espie GS, Krol M, Huner NPA, Bruce D (2006) Mechanism of the down regulation of photosynthesis by blue light in the cyanobacterium Synechocystis $\mathrm{sp}$ PCC 6803. BioChemistry 45(29):8952-8958
Six C, Thomas J-C, Garczarek L, Ostrowski M, Dufresne A, Blot N, Scanlan DJ, Partensky F (2007) Diversity and evolution of phycobilisomes in marine Synechococcus spp.: a comparative genomics study. Genome Biol 8(12):R259

Snellenburg JJ, Dekker JP, van Grondelle R, van Stokkum IHM (2013) Functional compartmental modeling of the photosystems in the Thylakoid Membrane at $77 \mathrm{~K}$. J Phys Chem B 117(38):11363-11371

Snellenburg JJ, Wlodarczyk LM, Dekker JP, van Grondelle R, van Stokkum IHM (2017) A model for the $77 \mathrm{~K}$ excited state dynamics in Chlamydomonas reinhardtii in state 1 and state 2. Biochim Biophys Acta(BBA)—Bioenerg 1858(1):64-72

Tian L, van Stokkum IHM, Koehorst RBM, Jongerius A, Kirilovsky D, van Amerongen H (2011) Site, rate, and mechanism of photoprotective quenching in cyanobacteria. J Am Chem Soc 133(45):18304-18311

Tian L, Gwizdala M, van Stokkum IHM, Koehorst RBM, Kirilovsky D, van Amerongen $\mathrm{H}$ (2012) Picosecond kinetics of light harvesting and photoprotective quenching in wild-type and mutant phycobilisomes isolated from the Cyanobacterium Synechocystis PCC 6803. Biophys J 102(7):1692-1700

Tian L, Farooq S, van Amerongen H (2013a) Probing the picosecond kinetics of the photosystem II core complex in vivo. Phys Chem Chem Phys 15(9):3146-3154

Tian L, van Stokkum IHM, Koehorst RBM, van Amerongen H (2013b) Light harvesting and blue-green light induced non-photochemical quenching in two different C-phycocyanin mutants of synechocystis PCC 6803. J Phys Chem B 117(38):11000-11006

van Grondelle R, Dekker JP, Gillbro T, Sundstrom V (1994) Energy transfer and trapping in photosynthesis. Biochim Biophys Acta(BBA)-Bioenerg 1187(1):1-65

van Stokkum IHM, Larsen DS, van Grondelle R (2004) Global and target analysis of time-resolved spectra. Biochim Biophys Acta 1657:82-104

Van Stokkum IHM, Van Oort B, Van Mourik F, Gobets B, Van Amerongen $\mathrm{H}$ (2008) (Sub)-Picosecond spectral evolution of fluorescence studied with a synchroscan streak-camera system and target analysis. In: Aartsma TJ, Matysik J (eds) Biophysical techniques in photosynthesis. Springer, Dordrecht, pp 223-240. doi: 10.1007/978-1-4020-8250-4_12

van Stokkum IHM, Desquilbet TE, van der Weij-de Wit CD, Snellenburg JJ, van Grondelle R, Thomas J-C, Dekker JP, Robert B (2013) Energy transfer and trapping in red-chlorophyll-free photosystem I from Synechococcus WH 7803. J Phys Chem B 117(38):11176-11183

van Stokkum IHM, Gwizdala M, Tian L, Snellenburg JJ, van Grondelle $\mathrm{R}$, van Amerongen H, Berera R (2017) A functional compartmental model of the Synechocystis PCC 6803 phycobilisome. Photosynth Res. doi: 10.1007/s11120-017-0424-5

Watanabe M, Ikeuchi M (2013) Phycobilisome: architecture of a lightharvesting supercomplex. Photosynth Res 116(2-3):265-276

Waterbury JB, Watson SW, Valois FW, Franks DG (1986) Biological and ecological characterization of the marine unicellular cyanobacterium Synechococcus. Can Bull Fish Aquat Sci 214:71-120

Wlodarczyk LM, Dinc E, Croce R, Dekker JP (2016) Excitation energy transfer in Chlamydomonas reinhardtii deficient in the PSI core or the PSII core under conditions mimicking state transitions. Biochim Biophys Acta (BBA)-Bioenerg 1857(6):625-633

Wyman M, Gregory RPF, Carr NG (1985) Novel role for phycoerythrin in a marine cyanobacterium, Synechococcus strain dc2. Science 230(4727):818-820

Xie J, Zhao J-q, Peng C (2002) Analysis of the disk-to-disk energy transfer processes in $\mathrm{C}$-phycocyanin complexes by computer simulation technique. Photosynthetica 40(2):251-257 